\title{
CHARACTER ANALOGUES OF INFINITE SERIES FROM A CERTAIN MODULAR TRANSFORMATION FORMULA
}

\author{
Sung-Geun Lim
}

ABSTRACT. In this paper, we find character analogues of infinite series identities which come from a certain modular transformation formula given by B. C. Berndt.

\section{Introduction}

A special case of the character analogues of Ramanujan-Guinand formula $[5,6]$ shows the following beautiful symmetric identity $[3] ;$

For $\alpha, \beta>0$ with $\alpha \beta=\pi^{2}$,

$$
\sum_{n=1}^{\infty}\left(\frac{n}{5}\right) \sigma_{-1}(n) e^{-2 \alpha n / 5}=\sum_{n=1}^{\infty}\left(\frac{n}{5}\right) \sigma_{-1}(n) e^{-2 \beta n / 5}
$$

where (:) means the Legendre symbol and

$$
\sigma_{s}(n)=\sum_{d \mid n} d^{s}
$$

This type of identities can be derived most naturally from the functional equation of the associated zeta-function, for example, see [4]. In this paper, we obtain this type of identities from character analogues of infinite series identities which come from Theorem 2.1 in [2].

The followings are necessary notation. We choose the branch of the argument as $-\pi \leq \arg w<\pi$ for a complex $w$. Let $e(w)=e^{2 \pi i w}$ and let $\mathbb{H}=\{\tau \in$ $\mathbb{C} \mid \operatorname{Im}(\tau)>0\}$, the upper half-plane. For every complex $\tau \in \mathbb{H}, V \tau=V(\tau)=$ $\frac{a \tau+b}{c \tau+d}$ denotes a modular transformation with $c>0$ and $c \equiv 0(\bmod N)$. Let $r=\left(r_{1}, r_{2}\right)$ and $h=\left(h_{1}, h_{2}\right)$ denote real vectors, and define the associated vectors $R$ and $H$ by

$$
R=\left(R_{1}, R_{2}\right)=\left(a r_{1}+c r_{2}, b r_{1}+d r_{2}\right)
$$

Received May 29, 2009; Revised February 12, 2010.

2010 Mathematics Subject Classification. 11F12, 34A25.

Key words and phrases. infinite series, modular transformation.

This work partially supported by BK21-Postech CoDiMaRo. 
and

$$
H=\left(H_{1}, H_{2}\right)=\left(d h_{1}-b h_{2},-c h_{1}+a h_{2}\right) .
$$

For a positive integer $N$, let $\lambda_{N}$ denote the characteristic function of the integers modulo $N$, i.e.,

$$
\lambda_{N}(m)= \begin{cases}1, & \text { if } m \equiv 0(\bmod N), \\ 0, & \text { otherwise. }\end{cases}
$$

For real $x, \alpha$ and $\operatorname{Re}(s)>1$, let

$$
\psi(x, \alpha, s):=\sum_{n+\alpha>0} \frac{e(n x)}{(n+\alpha)^{s}} .
$$

We see that the function $\psi(x, \alpha, s)$ can be analytically continued to the entire $s$-plane [1]. Let

$$
\Psi^{ \pm}(x, \alpha, s):=\psi(x, \alpha, s)+e\left( \pm \frac{s}{2}\right) \psi(-x,-\alpha, s) .
$$

For a real number $x,[x]$ denotes the greatest integer less than or equal to $x$ and $\{x\}:=x-[x]$. For $\tau \in \mathbb{H}$ and an arbitrary complex number $s$, we define

$$
A_{N}(\tau, s ; r, h):=\sum_{N m+r_{1}>0} \sum_{n-h_{2}>0} \frac{e\left(N m h_{1}+\left(\left(N m+r_{1}\right) \tau+r_{2}\right)\left(n-h_{2}\right)\right)}{\left(n-h_{2}\right)^{1-s}} .
$$

Let

$$
H_{N}(\tau, s ; r, h):=A_{N}(\tau, s ; r, h)+e\left(\frac{s}{2}\right) A_{N}(\tau, s ;-r,-h) .
$$

We now state the principal theorem which shall be used to obtain main results.

Theorem $1.1([2])$. Let $Q=\{\tau \in \mathbb{C} \mid \operatorname{Re}(\tau)>-d / c\}, \varrho_{N}=c\left\{R_{2}\right\}-N d\left\{R_{1} / N\right\}$ and $c=N c^{\prime}$. Then for $\tau \in Q$ and all $s$,

$$
\begin{aligned}
& (c \tau+d)^{-s} H_{N}(V \tau, s ; r, h) \\
= & H_{N}(\tau, s ; R, H)-\lambda_{N}\left(r_{1}\right) e\left(-r_{1} h_{1}\right)(c \tau+d)^{-s} \Gamma(s)(-2 \pi i)^{-s} \Psi^{+}\left(h_{2}, r_{2}, s\right) \\
& +\lambda_{N}\left(R_{1}\right) e\left(-R_{1} H_{1}\right) \Gamma(s)(-2 \pi i)^{-s} \Psi^{-}\left(H_{2}, R_{2}, s\right) \\
& +(2 \pi i)^{-s} L_{N}(\tau, s ; R, H),
\end{aligned}
$$

where

$$
\begin{aligned}
& L_{N}(\tau, s ; R, H) \\
:= & \sum_{j=1}^{c^{\prime}} e\left(-H_{1}\left(N j+N\left[R_{1} / N\right]-c\right)-H_{2}\left(\left[R_{2}\right]+1+\left[\left(N j d+\varrho_{N}\right) / c\right]-d\right)\right) \\
& \cdot \int_{C} u^{s-1} \frac{e^{-(c \tau+d)\left(N j-N\left\{R_{1} / N\right\}\right) u / c}}{e^{-(c \tau+d) u}-e\left(c H_{1}+d H_{2}\right)} \frac{e^{\left\{\left(N j d+\varrho_{N}\right) / c\right\} u}}{e^{u}-e\left(-H_{2}\right)} d u,
\end{aligned}
$$

where $C$ is a loop beginning at $+\infty$, proceeding in the upper half-plane, encircling the origin in the positive direction so that $u=0$ is the only zero of

$$
\left(e^{-(c \tau+d) u}-e\left(c H_{1}+d H_{2}\right)\right)\left(e^{u}-e\left(-H_{2}\right)\right)
$$


lying "inside" the loop, and then returning to $+\infty$ in the lower half plane. Here, we choose the branch of $u^{s}$ with $0<\arg u<2 \pi$.

Remark 1.2. Note that after evaluation of $L_{N}(\tau, s ; R, H)$ for an integer $s$, the transformation formula in Theorem 1.1 will be valid for all $\tau \in \mathbb{H}$ by analytic continuation.

Let $B_{n}(x)$ denote Bernoulli polynomials, $n \geq 0$, which are defined by

$$
\frac{t e^{x t}}{e^{t}-1}=\sum_{n=0}^{\infty} B_{n}(x) \frac{t^{n}}{n !}(|t|<2 \pi) .
$$

The $n$-th Bernoulli number $B_{n}, n \geq 0$, is defined by $B_{n}=B_{n}(0)$. Put $\bar{B}_{n}(x)=$ $B_{n}(\{x\}), n \geq 0$.

\section{A transformation formula for $\prod_{n=1}^{\infty}\left(1-q^{n}\right)^{-\chi(n)}$}

Let $\chi$ be a Dirichlet character of modulus $N$. Let

$$
f(q):=\prod_{n=1}^{\infty}\left(1-q^{n}\right)^{-\chi(n)},
$$

where $q=e(\tau)$ and $\tau \in \mathbb{H}$. Let $V q:=e(V \tau)$. In this section, we take a modular transformation $V$ as

$$
V \tau=\frac{\tau-1}{N \tau-N+1} .
$$

Theorem 2.1. Let $\chi$ be an even Dirichlet character of modulus $N$ and let $z=N \tau-N+1$. Then, for any $z \in \mathbb{H}$,

$$
\begin{aligned}
\log f(V q)= & \log f(q)-\pi i\left(z+z^{-1}\right) \sum_{k=1}^{\left[\frac{N-1}{2}\right]} \chi(k) B_{2}\left(\frac{k}{N}\right) \\
& +2 \pi i \sum_{k=1}^{\left[\frac{N-1}{2}\right]} \chi(k) B_{1}\left(\frac{k}{N}\right)^{2} .
\end{aligned}
$$

Proof. Since $\chi$ is even,

$$
\begin{aligned}
\log f(q) & =-\sum_{n=1}^{\infty} \chi(n) \log \left(1-q^{n}\right) \\
& =\sum_{n=1}^{\infty} \sum_{m=1}^{\infty} \chi(n) \frac{q^{n m}}{m} \\
& =\sum_{k=1}^{N-1} \chi(k) \sum_{n=0}^{\infty} \sum_{m=1}^{\infty} \frac{q^{(n N+k) m}}{m} \\
& =\sum_{k=1}^{\left[\frac{N-1}{2}\right]} \chi(k)\left(\sum_{n=0}^{\infty} \sum_{m=1}^{\infty} \frac{q^{(n N+k) m}}{m}+\sum_{n=0}^{\infty} \sum_{m=1}^{\infty} \frac{q^{(n N+N-k) m}}{m}\right)
\end{aligned}
$$




$$
=\sum_{k=1}^{\left[\frac{N-1}{2}\right]} \chi(k) H_{N}\left(\tau, 0 ; r_{k}, 0\right),
$$

where $r_{k}=(k, 0)$. Then, by Theorem 1.1, we have

$$
\begin{aligned}
\log f(V q) & =\sum_{k=1}^{\left[\frac{N-1}{2}\right]} \chi(k) H_{N}\left(V \tau, 0 ; r_{k}, 0\right) \\
& =\sum_{k=1}^{\left[\frac{N-1}{2}\right]} \chi(k)\left(H_{N}\left(\tau, 0 ; R_{k}, 0\right)+L_{N}\left(\tau, 0 ; R_{k}, 0\right)\right),
\end{aligned}
$$

where $R_{k}=(k,-k)$. It is easy to see that

$$
\begin{aligned}
H_{N}\left(\tau, 0 ; R_{k}, 0\right) & =\sum_{n=0}^{\infty} \sum_{m=1}^{\infty} \frac{q^{(n N+k) m}}{m}+\sum_{n=0}^{\infty} \sum_{m=1}^{\infty} \frac{q^{(n N+N-k) m}}{m} \\
& =H_{N}\left(\tau, 0 ; r_{k}, 0\right) .
\end{aligned}
$$

For $L_{N}\left(\tau, 0 ; R_{k}, 0\right)$, using the residue theorem, we obtain that

$$
\begin{aligned}
L_{N}\left(\tau, 0 ; R_{k}, 0\right) & =2 \pi i \sum_{j=0}^{2} \frac{B_{j}\left(1-\left\{\frac{k}{N}\right\}\right) \bar{B}_{2-j}\left(1+\frac{N k-k}{N}\right)}{j !(2-j) !}(-z)^{j-1} \\
& =2 \pi i \sum_{j=0}^{2} \frac{B_{j}\left(\frac{k}{N}\right) B_{2-j}\left(\frac{k}{N}\right)}{j !(2-j) !}(-z)^{j-1} \\
& =\pi i B_{2}\left(\frac{k}{N}\right)\left(z+z^{-1}\right)+2 \pi i B_{1}\left(\frac{k}{N}\right)^{2},
\end{aligned}
$$

where $z=N \tau-N+1$. Thus by combining (2.1), (2.2), (2.3) and applying Remark 1.2 , we obtain the desired result.

Let $\zeta_{N}=e^{2 \pi i / N}$ and let

$$
\sigma_{s}(\chi, n):=\sum_{d \mid n} \chi(d) d^{s} .
$$

Theorem 2.2. Let $\chi$ be an even Dirichlet character of modulus $N$. Then, for any $z \in \mathbb{H}$,

$$
\begin{aligned}
& \sum_{n=1}^{\infty} \chi(n) \zeta_{N}^{n} \sigma_{-1}(\bar{\chi}, n) e\left(-n z^{-1} / N\right) \\
= & \sum_{n=1}^{\infty} \chi(n) \zeta_{N}^{-n} \sigma_{-1}(\bar{\chi}, n) e(n z / N)-\pi i\left(z+z^{-1}\right) \sum_{k=1}^{\left[\frac{N-1}{2}\right]} \chi(k) B_{2}\left(\frac{k}{N}\right) \\
& +2 \pi i \sum_{k=1}^{\left[\frac{N-1}{2}\right]} \chi(k) B_{1}\left(\frac{k}{N}\right)^{2} .
\end{aligned}
$$


Proof. Since

$$
\begin{gathered}
V \tau=\frac{1}{N}\left(1-z^{-1}\right), \tau=\frac{1}{N}(z-1)+1, \\
e((n N+k) m V \tau)=\zeta_{N}^{k m} e\left(-(n N+k) m z^{-1} / N\right),
\end{gathered}
$$

$$
e((n N+k) m \tau)=\zeta_{N}^{-k m} e((n N+k) m z / N)
$$

we have

$$
\begin{aligned}
\log f(V q) & =\sum_{k=1}^{N-1} \chi(k) \sum_{n=0}^{\infty} \sum_{m=1}^{\infty} \zeta_{N}^{k m} \frac{e\left(-(n N+k) m z^{-1} / N\right)}{m} \\
& =\sum_{m=1}^{\infty} \frac{1}{m} \sum_{n=1}^{\infty} \chi(n) \zeta_{N}^{m n} e\left(-m n z^{-1} / N\right) \\
& =\sum_{n=1}^{\infty} \chi(n) \zeta_{N}^{n} \sigma_{-1}(\bar{\chi}, n) e\left(-n z^{-1} / N\right) .
\end{aligned}
$$

Similarly,

$$
\log f(q)=\sum_{n=1}^{\infty} \chi(n) \zeta_{N}^{-n} \sigma_{-1}(\bar{\chi}, n) e(n z / N) .
$$

Now, applying (2.6), (2.7) to Theorem 2.1, the proof is complete.

Theorem 2.3. Let $\alpha, \beta>0$ with $\alpha \beta=\pi^{2}$. Then

$$
\begin{aligned}
& \sum_{n=1}^{\infty} \chi(n) \zeta_{N}^{n} \sigma_{-1}(\bar{\chi}, n) e^{-2 \alpha n / N} \\
= & \sum_{n=1}^{\infty} \chi(n) \zeta_{N}^{-n} \sigma_{-1}(\bar{\chi}, n) e^{-2 \beta n / N}+(\beta-\alpha) \sum_{k=1}^{\left[\frac{N-1}{2}\right]} \chi(k) B_{2}\left(\frac{k}{N}\right) \\
& +2 \pi i \sum_{k=1}^{\left[\frac{N-1}{2}\right]} \chi(k) B_{1}\left(\frac{k}{N}\right)^{2} .
\end{aligned}
$$

Proof. Put $z=\frac{\pi i}{\alpha}$ in Theorem 2.2.

If $p$ is a prime with $p \equiv 1(\bmod 4)$, then we can put $\chi=(\dot{\bar{p}})$ in Theorem 2.3 , where $(\dot{\bar{p}})$ is the Legendre symbol.

Corollary 2.4. Let $p$ be a prime with $p \equiv 1(\bmod 4)$. Then

$$
\sum_{n=1}^{\infty}\left(\frac{n}{p}\right) \sigma_{-1}\left(\left(\frac{\cdot}{p}\right), n\right) \cos (2 \pi n / p) e^{-2 \alpha n / p}
$$




$$
=\sum_{n=1}^{\infty}\left(\frac{n}{p}\right) \sigma_{-1}\left(\left(\frac{\cdot}{p}\right), n\right) \cos (2 \pi n / p) e^{-2 \beta n / p}+(\beta-\alpha) \sum_{k=1}^{\frac{p-1}{2}}\left(\frac{k}{p}\right) B_{2}(k / p) .
$$

Proof. Let $\chi=(\dot{\bar{p}})$ in Theorem 2.3 and equate the real parts.

Corollary 2.5. Let $p$ be a prime with $p \equiv 1(\bmod 4)$. Then

$$
\begin{aligned}
& \sum_{n=1}^{\infty}\left(\frac{n}{p}\right) \sigma_{-1}\left(\left(\frac{\dot{p}}{p}\right), n\right) \sin (2 \pi n / p) e^{-2 \alpha n / p} \\
= & -\sum_{n=1}^{\infty}\left(\frac{n}{p}\right) \sigma_{-1}\left(\left(\frac{\dot{\bar{p}}}{p}\right), n\right) \sin (2 \pi n / p) e^{-2 \beta n / p}+2 \pi \sum_{k=1}^{\frac{p-1}{2}}\left(\frac{k}{p}\right) B_{1}(k / p)^{2} .
\end{aligned}
$$

Proof. Let $\chi=(\dot{\bar{p}})$ in Theorem 2.3 and equate the imaginary parts.

Corollary 2.4 and Corollary 2.5 do not show symmetric identities when they are compared with (1.1). However we obtain a generalized version of Theorem 2.3 and have some symmetric identities in next section.

\section{Generalized identities}

In this section, we obtain more generalized character analogues than Theorem 2.1

Theorem 3.1. Let $\chi$ be an even Dirichlet character of modulus $N$. Then for any integer $\ell$ and for any $z \in \mathbb{H}$,

$$
\begin{aligned}
& z^{2 \ell} \sum_{n=1}^{\infty} \chi(n) \zeta_{N}^{n} \sigma_{-2 \ell-1}(\bar{\chi}, n) e\left(-n z^{-1} / N\right) \\
= & \sum_{n=1}^{\infty} \chi(n) \zeta_{N}^{-n} \sigma_{-2 \ell-1}(\bar{\chi}, n) e(n z / N) \\
& +(2 \pi i)^{2 \ell+1} \sum_{k=1}^{\left[\frac{N-1}{2}\right]} \chi(k) \sum_{j=0}^{2 \ell+2} \frac{B_{j}\left(\frac{k}{N}\right) B_{2 \ell+2-j}\left(\frac{k}{N}\right)}{j !(2 \ell+2-j) !}(-z)^{j-1} .
\end{aligned}
$$

Proof. Let $r_{k}=(k, 0)$ and let

$$
V \tau=\frac{\tau-1}{N \tau-N+1} .
$$

We see that

$$
H_{N}\left(\tau,-2 \ell ; r_{k}, 0\right)=\sum_{m=0}^{\infty} \sum_{n=1}^{\infty} \frac{e((m N+k) n \tau)}{n^{1+2 \ell}}+\sum_{m=0}^{\infty} \sum_{n=1}^{\infty} \frac{e((m N+N-k) n \tau)}{n^{1+2 \ell}} .
$$


By Theorem 1.1,

$$
\begin{aligned}
& (N \tau-N+1)^{2 \ell} H_{N}\left(V \tau,-2 \ell ; r_{k}, 0\right) \\
= & H_{N}\left(\tau,-2 \ell ; R_{k}, 0\right)+(2 \pi i)^{2 \ell} L_{N}\left(\tau,-2 \ell ; R_{k}, 0\right),
\end{aligned}
$$

where $R_{k}=(k,-k)$. Multiplying both sides in (3.2) by $\chi(k)$ and summing over $k$, we find that

$$
\begin{aligned}
& (N \tau-N+1)^{2 \ell} \sum_{k=1}^{\left[\frac{N-1}{2}\right]} \chi(k) H_{N}\left(V \tau,-2 \ell ; r_{k}, 0\right) \\
= & \sum_{k=1}^{\left[\frac{N-1}{2}\right]} \chi(k) H_{N}\left(\tau,-2 \ell ; R_{k}, 0\right)+(2 \pi i)^{2 \ell} \sum_{k=1}^{\left[\frac{N-1}{2}\right]} \chi(k) L_{N}\left(\tau,-2 \ell ; R_{k}, 0\right) .
\end{aligned}
$$

Plugging (3.1) into (3.3),

$$
\begin{aligned}
& (N \tau-N+1)^{2 \ell} \sum_{k=1}^{N-1} \chi(k) \sum_{m=0}^{\infty} \sum_{n=1}^{\infty} \frac{e((m N+k) n V \tau)}{n^{1+2 \ell}} \\
= & \sum_{k=1}^{N-1} \chi(k) \sum_{m=0}^{\infty} \sum_{n=1}^{\infty} \frac{e((m N+k) n \tau)}{n^{1+2 \ell}} \\
& +(2 \pi i)^{2 \ell} \sum_{k=1}^{\left[\frac{N-1}{2}\right]} \chi(k) L_{N}\left(\tau,-2 \ell ; R_{k}, 0\right) .
\end{aligned}
$$

It comes from the residue theorem that

$$
L_{N}\left(\tau,-2 \ell ; R_{k}, 0\right)=2 \pi i \sum_{j=0}^{2 \ell+2} \frac{B_{k}\left(\frac{k}{N}\right) B_{2 \ell+k}\left(\frac{k}{N}\right)}{j !(2 \ell+2-j) !}(-N \tau+N-1)^{j-1} .
$$

Let $z=N \tau-N+1$. Apply (2.4), (2.5), (3.5) to (3.4) to obtain

$$
\begin{aligned}
& z^{2 \ell} \sum_{k=1}^{N-1} \chi(k) \sum_{m=0}^{\infty} \sum_{n=1}^{\infty} \zeta_{N}^{(m N+k) n} \frac{e\left(-(m N+k) n z^{-1} / N\right)}{n^{1+2 \ell}} \\
= & \sum_{k=1}^{N-1} \chi(k) \sum_{m=0}^{\infty} \sum_{n=1}^{\infty} \zeta_{N}^{-(m N+k) n} \frac{e((m N+k) n z / N)}{n^{1+2 \ell}} \\
& +(2 \pi i)^{2 \ell+1} \sum_{k=1}^{\left[\frac{N-1}{2}\right]} \chi(k) \sum_{j=0}^{2 \ell+2} \frac{B_{j}\left(\frac{k}{N}\right) B_{2 \ell+2-j}\left(\frac{k}{N}\right)}{j !(2 \ell+2-j) !}(-z)^{j-1} .
\end{aligned}
$$

Employing Remark 1.2, we obtain the desired results by the similar manner in (2.6).

If we put $\ell=0$ in Theorem 3.1, then Theorem 2.2 follows. 
Theorem 3.2. Let $\chi$ be an even Dirichlet character of modulus $N$. Let $\alpha, \beta>$ 0 with $\alpha \beta=\pi^{2}$. Then for any integer $\ell$,

$$
\begin{aligned}
& \alpha^{-\ell} \sum_{n=1}^{\infty} \chi(n) \zeta_{N}^{n} \sigma_{-2 \ell-1}(\bar{\chi}, n) e^{-2 \alpha n / N} \\
= & (-\beta)^{-\ell} \sum_{n=1}^{\infty} \chi(n) \zeta_{N}^{-n} \sigma_{-2 \ell-1}(\bar{\chi}, n) e^{-2 \beta n / N} \\
& -2^{2 \ell+1} \sum_{k=1}^{\left[\frac{N-1}{2}\right]} \chi(k) \sum_{j=0}^{2 \ell+2} \frac{B_{j}\left(\frac{k}{N}\right) B_{2 \ell+2-j}\left(\frac{k}{N}\right)}{j !(2 \ell+2-j) !}(-\pi i)^{j} \alpha^{1+\ell-j} .
\end{aligned}
$$

Proof. Put $z=\frac{\pi i}{\alpha}$ in Theorem 3.1.

Corollary 3.3. For any integer $M>0$,

$$
\begin{aligned}
& \alpha^{M} \sum_{n=1}^{\infty} \chi(n) \zeta_{N}^{n} \sigma_{2 M-1}(\bar{\chi}, n) e^{-2 \alpha n / N} \\
= & (-\beta)^{M} \sum_{n=1}^{\infty} \chi(n) \zeta_{N}^{-n} \sigma_{2 M-1}(\bar{\chi}, n) e^{-2 \beta n / N} .
\end{aligned}
$$

Proof. Let $\ell=-M$ for any integer $M>0$ in Theorem 3.2.

Corollary 3.3 does not look to show symmetric identities. But if we put $\chi=(\dot{p})$ for a prime $p$ with $p \equiv 1(\bmod 4)$, then we obtain moderately good symmetric identities.

Corollary 3.4. Let $p$ be a prime with $p \equiv 1(\bmod 4)$. Then for any integer $M>0$,

$$
\begin{aligned}
& \alpha^{2 M} \sum_{n=1}^{\infty}\left(\frac{n}{p}\right) \sigma_{4 M-1}\left(\left(\frac{\cdot}{p}\right), n\right) \cos (2 \pi n / p) e^{-2 \alpha n / p} \\
= & \beta^{2 M} \sum_{n=1}^{\infty}\left(\frac{n}{p}\right) \sigma_{4 M-1}\left(\left(\frac{\cdot}{p}\right), n\right) \cos (2 \pi n / p) e^{-2 \beta n / p} .
\end{aligned}
$$

Proof. Let $\chi=(\dot{p})$ in Corollary 3.3. Replace $M$ by $2 M$ and equate the real parts.

Corollary 3.5. Let $p$ be a prime with $p \equiv 1(\bmod 4)$. Then for any integer $M>0$,

$$
\begin{aligned}
& \alpha^{2 M-1} \sum_{n=1}^{\infty}\left(\frac{n}{p}\right) \sigma_{4 M-3}\left(\left(\frac{\cdot}{p}\right), n\right) \sin (2 \pi n / p) e^{-2 \alpha n / p} \\
= & \beta^{2 M-1} \sum_{n=1}^{\infty}\left(\frac{n}{p}\right) \sigma_{4 M-3}\left(\left(\frac{\cdot}{p}\right), n\right) \sin (2 \pi n / p) e^{-2 \beta n / p} .
\end{aligned}
$$


Proof. Let $\chi=(\dot{p})$ in Corollary 3.3. Replace $M$ by $2 M-1$ and equate the imaginary parts.

For an odd character $\chi$, by the same manner in case of even character, we have the following theorems and corollaries.

Theorem 3.6. Let $\chi(n)$ be an odd Dirichlet character of modulus $N$. Then for any integer $\ell$ and any $z \in \mathbb{H}$,

$$
\begin{aligned}
& z^{2 \ell+1} \sum_{n=1}^{\infty} \chi(n) \zeta_{N}^{n} \sigma_{-2 \ell-2}(\bar{\chi}, n) e\left(-n z^{-1} / N\right) \\
= & \sum_{n=1}^{\infty} \chi(n) \zeta_{N}^{-n} \sigma_{-2 \ell-2}(\bar{\chi}, n) e(n z / N) \\
& +(2 \pi i)^{2 \ell+2} \sum_{k=1}^{\left[\frac{N-1}{2}\right]} \chi(k) \sum_{j=0}^{2 \ell+3} \frac{B_{j}\left(\frac{k}{N}\right) B_{2 \ell+3-j}\left(\frac{k}{N}\right)}{j !(2 \ell+3-j) !}(-z)^{j-1} .
\end{aligned}
$$

Theorem 3.7. Let $\chi(n)$ be an odd Dirichlet character of modulus $N$. Let $\alpha, \beta>0$ with $\alpha \beta=\pi^{2}$. Then for any integer $\ell$,

$$
\begin{aligned}
& \alpha^{-\ell-\frac{1}{2}} \sum_{n=1}^{\infty} \chi(n) \zeta_{N}^{n} \sigma_{-2 \ell-2}(\bar{\chi}, n) e^{-2 \alpha n / N} \\
= & (-\beta)^{-\ell-\frac{1}{2}} \sum_{n=1}^{\infty} \chi(n) \zeta_{N}^{-n} \sigma_{-2 \ell-2}(\bar{\chi}, n) e^{-2 \beta n / N} \\
& +2^{2 \ell+2} \sum_{k=1}^{\left[\frac{N-1}{2}\right]} \chi(k) \sum_{j=0}^{2 \ell+3} \frac{B_{j}\left(\frac{k}{N}\right) B_{2 \ell+3-j}\left(\frac{k}{N}\right)}{j !(2 \ell+3-j) !}(-\pi i)^{j} \alpha^{\frac{3}{2}+\ell-j} .
\end{aligned}
$$

Corollary 3.8. For any integer $M>0$,

$$
\begin{aligned}
& \alpha^{M+\frac{1}{2}} \sum_{n=1}^{\infty} \chi(n) \zeta_{N}^{n} \sigma_{2 M}(\bar{\chi}, n) e^{-2 \alpha n / N} \\
= & (-\beta)^{M+\frac{1}{2}} \sum_{n=1}^{\infty} \chi(n) \zeta_{N}^{-n} \sigma_{2 M}(\bar{\chi}, n) e^{-2 \beta n / N} .
\end{aligned}
$$
3.7

If $p$ is a prime with $p \equiv 3(\bmod 4)$, then we can put $\chi=(\dot{\bar{p}})$ in Theorem

Corollary 3.9. Let $p$ be a prime with $p \equiv 3(\bmod 4)$. Then for any integer $M>0$,

$$
\alpha^{2 M+\frac{1}{2}} \sum_{n=1}^{\infty}\left(\frac{n}{p}\right) \sigma_{4 M}\left(\left(\frac{\cdot}{p}\right), n\right) \cos (2 \pi n / p) e^{-2 \alpha n / p}
$$




$$
=\beta^{2 M+\frac{1}{2}} \sum_{n=1}^{\infty}\left(\frac{n}{p}\right) \sigma_{4 M}\left(\left(\frac{\cdot}{p}\right), n\right) \sin (2 \pi n / p) e^{-2 \beta n / p} .
$$

Corollary 3.10. Let $p$ be a prime with $p \equiv 3(\bmod 4)$. Then for any integer $M>0$,

$$
\begin{aligned}
& \alpha^{2 M-\frac{1}{2}} \sum_{n=1}^{\infty}\left(\frac{n}{p}\right) \sigma_{4 M-2}\left(\left(\frac{\cdot}{p}\right), n\right) \cos (2 \pi n / p) e^{-2 \alpha n / p} \\
= & -\beta^{2 M-\frac{1}{2}} \sum_{n=1}^{\infty}\left(\frac{n}{p}\right) \sigma_{4 M-2}\left(\left(\frac{\cdot}{p}\right), n\right) \sin (2 \pi n / p) e^{-2 \beta n / p} .
\end{aligned}
$$

\section{References}

[1] B. C. Berndt, Two new proofs of Lerch's functional equation, Proc. Amer. Math. Soc. 32 (1972), 403-408.

[2] _ Analytic Eisenstein series, theta-functions, and series relations in the spirit of Ramanujan, J. Reine Angew. Math. 303/304 (1978), 332-365.

[3] B. C. Berndt, A. Dixit, and J. Sohn, Character analogues of formulas of Ramanujan, Koshliakov and Guinand, to appear in Adv. in Appl. Math.

[4] H. H. Chan, On the equivalence of Ramanujan's partition identities and a connection with the Rogers-Ramanujan continued fraction, J. Math. Anal. Appl. 198 (1996), no. 1, $111-120$.

[5] A. P. Guinand, Some rapidly convergent series for the Riemann $\xi$-function, Quart. J. Math. Oxford Ser. (2) 6 (1955), 156-160.

[6] S. Ramanujan, The Lost Notebook and Other Unpublished Papers, Narosa, New Delhi, 1988.

Department of Mathematics

Pohang University of Science and Technology

POHANG 790-784, Korea

E-mail address: sglimj@yahoo.co.kr 